\title{
Article
}

\section{Validation of a Commercial Loop-Mediated Isothermal Amplification (LAMP)-Based Kit for the Detection of Salmonella spp. According to ISO 16140:2016}

\author{
Calogero Di Bella ${ }^{1}\left({ }^{\circ}\right.$, Antonella Costa ${ }^{2}$, Sonia Sciortino ${ }^{2}$, Giuseppa Oliveri ${ }^{2}$, Gaetano Cammilleri ${ }^{3}$, \\ Francesco Geraci ${ }^{1}$, Daniela Lo Monaco ${ }^{1}$, Davide Carpintieri ${ }^{1}$, Giuseppe Lo Bue ${ }^{1}$, Carmelo Bongiorno ${ }^{4}$, \\ Alessandro Altomare ${ }^{1}$, Valentina Ciprì ${ }^{1, * \mathbb{C}}$, Rosario Pitti ${ }^{5}$, Carmine Lanzillo ${ }^{5}$, Giuseppe Arcoleo ${ }^{5}$ \\ and Rosalinda Allegro ${ }^{1}$ (1)
}

check for

updates

Citation: Di Bella, C.; Costa, A.; Sciortino, S.; Oliveri, G.; Cammilleri,

G.; Geraci, F.; Lo Monaco, D.;

Carpintieri, D.; Lo Bue, G.; Bongiorno, C.; et al. Validation of a Commercial

Loop-Mediated Isothermal Amplification (LAMP)-Based Kit for the Detection of Salmonella spp. According to ISO 16140:2016. Appl. Sci. 2021, 11, 6669. https://doi.org/ 10.3390/app11156669

Academic Editors: Francesco

Cappello and Magdalena

Gorska-Ponikowska

Received: 23 June 2021

Accepted: 19 July 2021

Published: 21 July 2021

Publisher's Note: MDPI stays neutral with regard to jurisdictional claims in published maps and institutional affiliations.

Copyright: (c) 2021 by the authors. Licensee MDPI, Basel, Switzerland. This article is an open access article distributed under the terms and conditions of the Creative Commons Attribution (CC BY) license (https:// creativecommons.org/licenses/by/ $4.0 /)$.
1 Area Sorveglianza Epidemiologica, Istituto Zooprofilattico Sperimentale della Sicilia (IZSSi), Via G. Marinuzzi 3, 90129 Palermo, Italy; calogero.dibella@izssicilia.it (C.D.B.); francesco.geraci@izssicilia.it (F.G.); daniela.lomonaco@izssicilia.it (D.L.M.); da.car19@gmail.com (D.C.);

lobuegiuseppe1981izs@gmail.com (G.L.B.); alessandro.altomare@izssicilia.it (A.A.); rosalinda.allegro@gmail.com (R.A.)

2 Area Microbiologia degli Alimenti, Istituto Zooprofilattico Sperimentale della Sicilia (IZSSi), Via G. Marinuzzi 3, 90129 Palermo, Italy; antonella.costa@izssicilia.it (A.C.); sonia.sciortino@izssicilia.it (S.S.); giuseppa.oliveri@izssicilia.it (G.O.)

3 Dipartimento Alimenti, Area Chimica e Tecnologie Alimentari, Istituto Zooprofilattico Sperimentale della Sicilia (IZSSi), Via G. Marinuzzi 3, 90129 Palermo, Italy; gaetano.cammilleri@izssicilia.it

4 Area Affari Generali Legali e Contenzioso, Istituto Zooprofilattico Sperimentale della Sicilia (IZSSi), Via G. Marinuzzi 3, 90129 Palermo, Italy; carmelo.bongiorno@izssicilia.it

5 Enbiotech s.r.l. Via Aquileia 34, 90144 Palermo, Italy; r.pitti@enbiotech.eu (R.P.); c.lanzillo@enbiotech.eu (C.L.); g.arcoleo@enbiotech.eu (G.A.)

* Correspondence: valentinacipri@gmail.com; Tel.: +39-3201-816-981

Featured Application: The validated LAMP kit provides an accurate method for the rapid detection of Salmonella spp., offering significant advantages over the traditional method, as it is characterised by a high sensitivity, easiness of use for laboratory testing, and a large reduction in the analysis time, making it a valuable asset to the food industry.

Abstract: The traditional cultural method (PCR and Real-Time PCR) for Salmonella spp. detection and identification is laborious and time-consuming. A qualitative LAMP method detecting Salmonella spp. was validated in compliance with ISO 16140:2016. The results show a relative accuracy, sensitivity, and specificity of $100 \%$ in comparison with the reference method ISO 6579-1:2017; the LOD50 was set as $0.4 \mathrm{CFU} / \mathrm{g}$. Additionally, a field study was carried out comparing the LAMP kit, a commercially available Real-Time PCR kit (FoodProof Salmonella, Biotecon Diagnostics), and the reference cultural method. The Salmonella spp. LAMP kit was suitable for reliable detection of Salmonella spp., simplifying and reducing the extent and the steps of the analytical process. A total of 105 samples of raw poultry meat were screened for the presence of Salmonella spp. according to three methods: the LAMP kit Salmonella spp. (Enbiotech), the Real-Time PCR kit FoodProof Salmonella (Biotecon), and the reference cultural method. Using these three methods, only one sample out of the $105(0.95 \%)$ tested was positive for Salmonella spp. This sample was further investigated using the reference method described in ISO 6579-3:2014, in order to characterise the Salmonella strain. Following this further biochemical identification and serological typing, the isolate was characterised as Salmonella Infantis.

Keywords: LAMP; Salmonella; ISO 16140:2016; food-borne; validation; specificity; sensitivity; accuracy; kit 


\section{Introduction}

Salmonella is a highly relevant food-borne pathogen of large economic significance for both animals and humans. Salmonella outbreaks caused 94,530 human cases in the EU only in 2016, with the highest burden relating to the number of hospitalizations (1766) and deaths (10) [1]. S. enteritidis, in particular, accounted for $59 \%$ of all Salmonella infection cases originating in the EU. The principal reservoirs are the intestines of a wide variety of animals, resulting in the contamination of different foodstuffs, both of animal and plant origin [2,3]. Indeed, Salmonella is associated mainly with raw food, subject to faecal contamination, including poultry, raw meat, seafood, egg, and dairy products [4]. In addition, it was also found to be the most common bacterial pathogen responsible for produce outbreaks [5].

Therefore, in EU countries, surveillance of Salmonella infections in humans is compulsory, and also for food-producing animals and food thereof. In order to guarantee food security, the availability of reliable methods to identify this pathogenic bacterium is becoming increasingly relevant to the food industry, as well as for official controls.

The traditional cultural method [6] requires more than five days to determine a positive result, besides being laborious and time-consuming. For this reason, to use the traditional cultural method is not very suitable for high-throughput screening of large numbers of food samples for the presence of Salmonella cells $[7,8]$. Less laborious and faster alternative methods for pathogen detection in foods have been developed [8-12]. Among them, the method that combines loop-mediated isothermal amplification (LAMP) with bioluminescence detection stands out as a reliable, faster, and simpler approach than conventional culture methods. LAMP was developed by Notomi et al. in 2000 [9]. It is a method that amplifies DNA with high specificity, efficiency, and rapidity, utilizing a DNA polymerase enzyme with high strand displacement activity and two pairs of primers recognizing six independent sequences of a target gene under isothermal conditions [12]. Subsequently this method has been implemented by Nagamine et al. in 2002, incorporating forward loop primers that accelerate the LAMP reaction and reducing costs as a consequence [13]. Due to its high sensitivity and low cost, LAMP has been applied for pathogen detection screening of large numbers of food samples, and has successfully been used to detect many pathogens, including Salmonella spp. [14]. In recent years, some kits based on LAMP have been commercialised and their performance has been positively evaluated [8,12]. Hence alternative methods are catching on, as molecular methods are more rapid and have an interesting potential to be used for screening, revealing a preliminary result, even if ISO 6579-1:2017 [6] still remains necessary to isolate the microorganisms for further characterization. ISO 16140-2:2016 [15] defines the procedures for validation of alternative microbiological methods against the cultural method, measuring the concordance of the results for both methods.

A Salmonella LAMP (loop-mediated isothermal amplification) assay was validated in this study as a novel specific and cost-effective nucleic acid amplification method for bacterial detection and identification. This innovative method is characterised by six primers that specifically recognise eight different regions on the target gene [9].

In comparison to PCR and Real-Time PCR, LAMP has many advantages: reaction simplicity, as it can be performed by semi-skilled staff, even in a heating block without any thermal cycler; and detection sensitivity, displaying a 10-100-fold higher sensitivity than PCR [16]. In addition, LAMP shows a higher amplification efficiency and the enzyme commonly involved, Bst DNA polymerase, has shown not to be inhibited by the presence of anticoagulants, $\mathrm{NaCl}$, hemin, and other PCR-interfering substances [17]. Due to its simplicity, the LAMP technique has initially been applied to diagnosis, but recently it has also been extended to genetically modified organisms and identification of meat and fish species in food products [18]. For these reasons, it represents an ideal candidate for point-of-care diagnosis and when rapid results are needed, such as in food industry, where Salmonella spp. positive samples need to be immediately blocked for public health and safety. 
In this study, we evaluated the LAMP method through validating the kit by comparing it to different methods that are currently the most commonly used.

\section{Materials and Methods}

All the processed samples used for the method optimisation and validation came from a large-scale distribution, in order to reduce any bias from local food specialities and extend the range of the validation. Samples were chosen as positive samples for the validation of the method and for matrix effects evaluation. All the food samples came from Italian supermarkets.

\subsection{DNA Extraction Genomic}

Several samples were tested, such as heat-processed milk and dairy products, raw poultry and ready-to-cook poultry products, eggs and egg products (derivate), ready-to-eat and ready-to-reheat fishery products, as well as fresh produce and fruits.

The kit Salmonella spp. (Enbiotech) provides a rapid preliminary DNA extraction from food matrices. In the pre enrichment phase, $25 \mathrm{~g}$ of a sample was taken and homogenated with $225 \mathrm{~mL}$ of Buffered Peptone Water (BPW). After a $22 \pm 2 \mathrm{~h}$ enrichment in Buffered Peptone Water (BPW) at $37^{\circ} \mathrm{C}$.

The DNA extraction was performed using a ready-to-use buffer contained in the Salmonella Screen Glow kit (Enbiotech). Then, $250 \pm 50 \mathrm{mg}$ of a sample was directly placed into $15 \mathrm{~mL}$ tubes containing $4 \mathrm{~mL}$ of the ready-to-use extraction buffer (Enbiotech) and then incubated for $40 \pm 5 \mathrm{~min}$ at room temperature.

Genetic amplification using LAMP technology and real-time detection of the results using the dedicated device ICGENE mini (Enbiotech). The kit is ready-to-use and includes:

- DNA extraction buffer, through chemical lysis;

- Tubes strip containing lyophilised primers;

- Amplification master mix;

- Mineral oil;

- Positive control;

- Negative control;

- Sterile water.

\subsection{LAMP Assays}

The analytical and diagnostic assays to recognise Salmonella spp. DNA was performed using the Salmonella Screen Glow commercial kit (Enbiotech) with an ICGENE mini portable instrument (Enbiotech), consisting of a real-time fluorimeter, monitored and regulated by the the ICGENE application (Enbiotech), and downloadable on various smart devices. The Salmonella Screen Glow commercial kit includes ready-to-use reaction tubes (containing primers, fluorescent dye, etc.) to achieve a rapid amplification of the DNA template. The protocol to obtain the specific amplification of the target Salmonella spp. DNA was carried out in a mixture with a final volume of $55 \mu \mathrm{L}$, including $22 \mu \mathrm{L}$ of the Salmonella Screen Glow LAMP mix (Enbiotech), $30 \mu \mathrm{L}$ of mineral oil, and $3 \mu \mathrm{L}$ of the extracted DNA samples. The mineral oil was added to the top of the reaction mixture to prevent evaporation. The amplification was optimised and performed at $65^{\circ} \mathrm{C}$ for $35 \mathrm{~min}$. Realtime monitoring of the fluorescence associated with the amplification was possible using the fluorimeter of the ICGENE portable instrument and the ICGENE application interface.

\subsection{Validation Plan}

The study was carried out at the Food Microbiology Laboratory of the Istituto Zooprofilattico Sperimentale of Sicily, Palermo (Italy), according to the validation process explained by ISO 16140-2:2016 [15]. According to ISO 16140, we demonstrated that the results obtained with the alternative method (LAMP kit) were comparable (at least equivalent) to the results obtained with the reference method. Following the validation protocol described by ISO 16140-2:2016 [15], we did a comparative study of the alternative method with the 
corresponding reference method, conducted by the Food Microbiology Laboratory of the Istituto Zooprofilattico Sperimentale of Sicily, Palermo (Italy). Validation determined the following parameters: relative limit of detection (RLOD), inclusivity, exclusivity and a method comparison study including relative accuracy (AC), relative specificity (SP), and sensitivity (SE). Examining the samples both with the alternative (LAMP kit) method and the reference method, the parameters of AC, SP, and SE were calculated as follows:

$$
\begin{gathered}
\mathrm{AC}=(\mathrm{PA}+\mathrm{NA}) / \mathrm{N} \times 100 \% \\
\mathrm{SP}=(\mathrm{NA}) / \mathrm{N}_{-} \times 100 \% \\
\mathrm{SE}=(\mathrm{PA}) / \mathrm{N}_{+} \times 100 \%
\end{gathered}
$$

where $\mathrm{PA}=$ positive agreement; $\mathrm{NA}=$ negative agreement; $\mathrm{N}=$ total number of samples; $\mathrm{N}_{-}=$number of negative samples; and $\mathrm{N}_{+}=$number of positive samples.

The RLOD was calculated as follows:

$$
\text { RLOD }=\text { LOD50 alternative method/LOD50 reference method }
$$

where LOD50 = the limit of detection (LOD) of 50\% = the smallest amount of analyte that can be detected but not quantified with a 50\% probability. Therefore, LOD50 is the level of detection for which $50 \%$ of tests give a positive result [19].

RLOD, SP, and SE were performed against the reference method ISO 6579-1:2017 [6] "Microbiology of the food chain-Horizontal method for the detection, enumeration and serotyping of Salmonella-Part 1: Detection of Salmonella spp."

\subsection{Bacterial Strains}

Bacterial strains were maintained on cryogenic beads at $-20^{\circ} \mathrm{C}$; before use, the beads were placed in Columbia Blood Agar (CBA-Microbiol) plates and incubated at $37^{\circ} \mathrm{C}$ for 18-24 h. Subsequently, the bacterial strains were placed in Tryptone Soya Agar (TSAMicrobiol) tubes, incubated at $37^{\circ} \mathrm{C}$ for $18-24 \mathrm{~h}$ and maintained at $4{ }^{\circ} \mathrm{C}$ for two weeks. For RLOD and the comparative studies, a strain of Salmonella enteritidis ATCC 13,076 was used. For the inclusivity study, field strains cultures coming from food samples were used, which were identified and confirmed by ISO/TR 6579-3:2014 [20].

Inclusivity of the LAMP method was evaluated by testing 25 pure cultures of the target microorganisms, while exclusivity was determined by testing 30 pure cultures of species other than Salmonella spp. (Table 1).

A comparative study was performed comparing the LAMP kit and the reference method. In particular, this study allowed evaluating the relative accuracy, relative specificity, and sensitivity. Food samples were chosen based on the categories given in Table A1 of ISO 16140-2:2016 [15].

In particular, five categories were chosen among the most relevant:

- Heat-processed milk and dairy products;

- Raw poultry and ready-to-cook poultry products;

- $\quad$ Eggs and egg products (derivate);

- Ready-to-eat, ready-to-reheat fishery products;

- Fresh produce and fruits.

For each category, 60 samples were tested, made up of 3 specific typologies with 20 samples representative of each typology ( 3 typologies $\times 20$ samples for each $=60$ samples per category). Of tested samples per typology, $50 \%$ (i.e., 10 ) were negative and $50 \%$ were spiked and hence positive.

RLOD tests were run on the same five food matrices of the comparative study: heatprocessed milk and dairy products; raw poultry and ready-to-cook poultry products; eggs and egg products (derivates); ready-to-eat, ready-to-reheat fishery products; and fresh produce and fruits. 
Table 1. Salmonella spp. tested for inclusivity and species used for exclusivity testing.

\begin{tabular}{|c|c|c|c|c|}
\hline \multicolumn{5}{|c|}{ Microorganism } \\
\hline & Tested for Inclusivity & & Species Used for Exclusivity Testing & Code \\
\hline 1 & S. Livingstone & 1 & Aeromonas hydrophila & ATCC 35650 \\
\hline 2 & S. Heron & 2 & Arcobacter butzleri & NCTC 12481 \\
\hline 3 & S. Corn & 3 & Bacillus cereus & ATCC 11778 \\
\hline 4 & S. Madelia & 4 & Bacillus cereus & B25052 \\
\hline 5 & S. Typhimurium (monophasic) & 5 & Bacillus subtilis & BCS51 \\
\hline 6 & S. Thompson & 6 & Campylobacter coli & ATCC 33559 \\
\hline 7 & S. Virchow & 7 & Campylobacter jejuni & ATCC 33291 \\
\hline 8 & S. London & 8 & Citrobacter freudii & ATCC 8990 \\
\hline 9 & S. Typhimurium & 9 & Clostridium bifermentans & CBIF107 \\
\hline 10 & S. Kissi & 10 & Clostridium perfringens & ATCC 13124 \\
\hline 11 & S. Blocklei & 11 & Escherichia coli & ATCC 25922 \\
\hline 12 & S. Toulon & 12 & Escherichia coli 0157 & ATCC 35150 \\
\hline 13 & S. Halle & 13 & Enterobacter cloacae & Not available \\
\hline 14 & S. Abony & 14 & Enterococcus faecium & EFC49 \\
\hline 15 & S. Messina & 15 & Enterobacter sakazakii & ATCC 29544 \\
\hline 16 & S. Montevideo & 16 & Listeria innocua & ATCC 33090 \\
\hline 17 & S. Potsdam & 17 & Listeria ivanovii & ATCC 19119 \\
\hline 18 & S. Muenster & 18 & Listeria monocytogenes & ATCC 7684 \\
\hline 19 & S. Larochelle & 19 & Listeria seeligeri & Not available \\
\hline 20 & S. Newport & 20 & Micrococcus luteus & ATCC 9341 \\
\hline 21 & S. Hadar & 21 & Psudomonas aeruginosa & ATCC 10145 \\
\hline 22 & S. Poona & 22 & Rhodococcus equi & ATCC 6939 \\
\hline 23 & S. Muenchen & 23 & Staphylococcus aureus & ATCC 25923 \\
\hline 24 & S. Derby & 24 & Staphylococcus aureus & ATCC 38862 \\
\hline 25 & S. Kottbus & 25 & Shigella sonnei & ATCC 9290 \\
\hline & & 26 & Streptococccus agalactiae & STRA41 \\
\hline & & 27 & Vibrio cholerae & ATCC 1473A \\
\hline & & 28 & Vibrio parahaemolyticus & ATCC 17802 \\
\hline & & 29 & Vibrio vulnificus & ATCC 27562 \\
\hline & & 30 & Yersinia enterocolitica & ATCC 23715 \\
\hline
\end{tabular}

From TSA, Salmonella enteritidis was inoculated in Xylose Lysine Deoxycholate agar (XLD-Microbiol) plates to obtain isolated colonies. XLD agar plates were incubated at $37^{\circ} \mathrm{C}$ for $24 \mathrm{~h}$.

In order to calculate the RLOD value, each of the five categories was spiked with the target microorganisms at three levels of contamination. In particular, they were 5 replicates of negative samples $(0 \mathrm{CFU}), 20$ replicates of the lowest detection level $(0.4 \mathrm{CFU} / \mathrm{g})$, and 5 replicates of a higher contamination level $(4 \mathrm{CFU} / \mathrm{g})$, for a total of 30 contaminated samples for each food category. In total, $25 \mathrm{~g}$ of each the different food samples were inoculated with the corresponding level of contamination and the samples were then stabilized at room temperature or $4{ }^{\circ} \mathrm{C}$, depending on food typology and its storage temperature. Subsequently, the reference and the alternative methods were performed.

\section{Results}

The method was optimised for the DNA extraction phase by testing in triplicate the initial weight of the samples at $250 \mathrm{mg}$. The extract was tested with three levels of contamination: $0,0.4$, and $4 \mathrm{CFU} / \mathrm{g}$.

\subsection{Validation}

As for inclusivity and exclusivity, the results showed all samples were correctly recognised; as a matter of fact, all target microorganisms were identified, while the relevant range of other species tested did not interfere. Table 2.

The relative accuracy, specificity, and sensitivity of each food category are reported in 
Table 2. Relative accuracy, relative sensitivity, and relative specificity of the alternative method (Salmonella spp.).

\begin{tabular}{|c|c|c|c|c|c|c|c|c|}
\hline Category & PA & NA & PD & ND & $\mathbf{N}$ & AC & SE & SP \\
\hline Heat-processed milk and dairy products & 30 & 30 & 0 & 0 & 60 & $100 \%$ & $100 \%$ & $100 \%$ \\
\hline Raw poultry and ready-to-cook poultry products & 30 & 30 & 0 & 0 & 60 & $100 \%$ & $100 \%$ & $100 \%$ \\
\hline Eggs and egg products (derivates) & 30 & 30 & 0 & 0 & 60 & $100 \%$ & $100 \%$ & $100 \%$ \\
\hline Fresh produce and fruits & 30 & 30 & 0 & 0 & 60 & $100 \%$ & $100 \%$ & $100 \%$ \\
\hline Ready-to-eat, ready-to-reheat fishery products & 30 & 30 & 0 & 0 & 60 & $100 \%$ & $100 \%$ & $100 \%$ \\
\hline Heat-processed milk and dairy products & 30 & 30 & 0 & 0 & 60 & $100 \%$ & $100 \%$ & $100 \%$ \\
\hline Raw poultry and ready-to-cook poultry products & 30 & 30 & 0 & 0 & 60 & $100 \%$ & $100 \%$ & $100 \%$ \\
\hline Eggs and egg products (derivates) & 30 & 30 & 0 & 0 & 60 & $100 \%$ & $100 \%$ & $100 \%$ \\
\hline Fresh produce and fruits & 30 & 30 & 0 & 0 & 60 & $100 \%$ & $100 \%$ & $100 \%$ \\
\hline Ready-to-eat, ready-to-reheat fishery products & 30 & 30 & 0 & 0 & 60 & $100 \%$ & $100 \%$ & $100 \%$ \\
\hline Heat-processed milk and dairy products & 30 & 30 & 0 & 0 & 60 & $100 \%$ & $100 \%$ & $100 \%$ \\
\hline
\end{tabular}

The results showed $100 \%$ for all three parameters for all the food categories; hence, neither false-positive nor false-negative samples were detected, with this $100 \%$ performance consistent with the reference method.

The RLOD value obtained was 1 for all the categories; therefore, the same LOD was reached both for the LAMP kit and for the reference method, while the detection limit was set at $0.4 \mathrm{CFU} / \mathrm{g}$ for all food categories (Table 3). At this concentration, false-negative results were found with the LAMP method with the commercialised kit: a sample of the heat-processed milk and dairy products and three samples of raw poultry and ready-tocook poultry products (Table 3). For the detection limit of $0.4 \mathrm{CFU} / \mathrm{g}$, the alternative (LAMP kit) method proved to be less sensitive than the traditional ones.

Table 3. Data for RLOD calculation (N. tot = total number of samples; N. pos ref = number of positives with the reference method; N. pos kit = number of positives with the Salmonella spp.).

\begin{tabular}{|c|c|c|c|c|c|}
\hline Category & Contamination Level & CFU/g & N. Tot & N. Pos Ref & N. Pos Kit \\
\hline Heat-processed & 1 & 0 & 5 & 0 & 0 \\
\hline milk and dairy & 2 & 0.4 & 20 & 20 & 19 \\
\hline products & 3 & 4 & 5 & 5 & 5 \\
\hline \multirow{3}{*}{$\begin{array}{l}\text { Raw poultry and } \\
\text { ready-to-cook } \\
\text { poultry products }\end{array}$} & 1 & 0 & 5 & 0 & 0 \\
\hline & 2 & 0.4 & 20 & 20 & 17 \\
\hline & 3 & 4 & 5 & 5 & 5 \\
\hline \multirow{3}{*}{$\begin{array}{c}\text { Eggs and egg } \\
\text { products } \\
\text { (derivates) }\end{array}$} & 1 & 0 & 5 & 0 & 0 \\
\hline & 2 & 0.4 & 20 & 20 & 20 \\
\hline & 3 & 4 & 5 & 5 & 5 \\
\hline \multirow{3}{*}{$\begin{array}{l}\text { Fresh produce and } \\
\text { fruits }\end{array}$} & 1 & 0 & 5 & 0 & 0 \\
\hline & 2 & 0.4 & 20 & 20 & 20 \\
\hline & 3 & 4 & 5 & 5 & 5 \\
\hline \multirow{3}{*}{$\begin{array}{l}\text { Ready-to-eat, } \\
\text { ready-to-reheat } \\
\text { fishery products }\end{array}$} & 1 & 0 & 5 & 0 & 0 \\
\hline & 2 & 0.4 & 20 & 20 & 20 \\
\hline & 3 & 4 & 5 & 5 & 5 \\
\hline
\end{tabular}

\subsection{Field Study}

A total of 105 samples of raw poultry meat were screened for the presence of Salmonella spp. according to three methods: the LAMP kit Salmonella spp. (Enbiotech), the Real-Time PCR kit FoodProof Salmonella (Biotecon), and the reference cultural method. Using these three methods, only one sample out of the $105(0.95 \%)$ tested was positive for Salmonella spp. This sample was further investigated using the reference method described in ISO. 


\section{Discussion}

In recent years, different diagnostic approaches have been developed for detecting various food pathogens, including innovative molecular methods.

Salmonella infections have been declining constantly since the implementation of EU control measures in poultry in 2007, although the data for 2016 showed a relevant increase of $11.5 \%$ in the number of cases compared to the previous year [1], underlining the need for continued risk management plans both at the state and at the food industry level.

In this context, the use of alternative methods, such as the "Salmonella spp." kit that can rapidly identify pathogenic bacteria, is of great relevance, provided they are validated against the standardized reference method as stated by ISO 16140-2:2016 [15].

The results obtained by the validated method in the comparative studies were equivalent to the microbiological reference method, hence providing a valid alternative to the cultural method. The relative sensitivity was found to be $100 \%$ for all the food typologies examined, confirming the absence of inhibition by different kinds of substrates. Moreover, the kit is characterised by a $100 \%$ specificity, as it does not amplify the other species tested, and it is inclusive of at least 25 serovars of Salmonella evaluated. At the detection limit of $0.4 \mathrm{CFU} / \mathrm{g}$, the LAMP kit showed false-negative results for 4 out of 100 samples; but, even though there is a lower sensitivity than the traditional methods, it still has the advantage in terms of speed of execution and ease of use.

During this study, we also compared the LAMP kit with another commercially available diagnostic method: Real-Time PCR in raw poultry samples. The results indicate that all the methods were in good agreement, even if a limitation of this study is the scarcity of positive results (i.e., 1 sample) that could hinder a more deepened evaluation. No problems of PCR inhibition were found using the internal amplification control provided in the kit; hence, in negative samples, the absence of pathogenic microorganisms was effectively determined.

Another feature of this study is that out of the 105 poultry samples screened for the presence of Salmonella spp., only 1 positive sample occurred, with a prevalence of $0.95 \%$. Samples were bought in different retail markets in order to have a more realistic representation of Sicilian poultry contamination. In fact, poultry flocks, particularly chicken, are frequently colonized with Salmonella without any detectable symptoms by horizontal and vertical transmission at the primary production level [21].

In European countries, the percentage of Salmonella-positive samples from fresh broiler meat is quite higher (4.85\%) [22], even if, besides retail, also samples from slaughterhouse and processing plants are included.

Although our prevalence is rather low, this should be taken as an additional motivation for the continuous control of this pathogens, as an effective implementation of control measures could still decrease the prevalence, producing safer food. Constant monitoring is mandatory to avoid new difficulties, such as the increasing antibiotic resistance in Salmonella spp. that has become a severe issue for public health at a global level [23].

\section{Conclusions}

The data in this study support the suitability of the Salmonella spp. kit for commercial use on different food samples, including egg products and poultry meat, which are the foods most associated with salmonellosis.

Therefore, the validated LAMP kit provides an accurate method for the rapid detection of Salmonella spp., offering significant advantages over the traditional method, as it is characterised by a high sensitivity (up to $0.4 \mathrm{CFU} / \mathrm{g}$ ), easiness of use for laboratory testing, and a large reduction in the analysis time (about $26 \mathrm{~h}$ to obtain definitive results), making it a valuable asset to the food industry. Despite the LAMP kit being less sensitive than the traditional methods, the great rapidity and ease of use suggest that the LAMP assay can be a valid alternative for routine examination in the food sector and for screening of large numbers of food samples. 
In our study we isolated the $S$. Infantis strain from the positive sample. In the EU, an increased occurrence of various serotypes implicated in human infections, including $S$. Infantis, has been reported, related to poultry meat [24]. The increase in $S$. Infantis has been associated with the propagation of various clones of broiler origin in different European countries, including the dominant Hungarian clone [25].

Continuous monitoring to detect Salmonella along the food chain is of critical importance for public health, above all in the poultry meat industry, as poultry meat is one of the most consumed meats globally and thus one of the most traded meat products.

Author Contributions: Conceptualization, V.C. and C.D.B.; methodology, V.C., A.C., S.S., G.O., D.C., R.P., C.L. and G.A.; software, G.L.B.; validation, F.G.; formal analysis, R.A.; investigation, G.C. and A.A.; resources, C.D.B.; data curation, R.A.; writing-original draft preparation, V.C.; writingreview and editing, V.C. and D.L.M.; supervision, C.D.B.; project administration, C.B. All authors have read and agreed to the published version of the manuscript.

Funding: This research received no external funding.

Institutional Review Board Statement: Not applicable.

Informed Consent Statement: Not applicable.

Data Availability Statement: The raw data supporting the conclusions of this article will be made available by the authors, without undue reservation, to any qualified researcher.

Acknowledgments: We wish to thank our colleagues involved in this scientific collaboration at the Istituto Zooprofilattico Sperimentale della Sicilia (IZSSi).

Conflicts of Interest: The authors declare that the research was conducted in the absence of any commercial or financial relationships that could be construed as a potential conflict of interest. In the interest of transparency, it should be pointed out that authors R.P., C.L. and G.A. are employed by Enbiotech srl. They offered only a methodological background contribution but had no role in the experimentation; in the design of the study; in the collection, analyses, or interpretation of data; in the writing of the manuscript; or in the decision to publish the results.

\section{References}

1. European Food Safety Authority (EFSA). The European Union summary report on trends and sources of zoonoses, zoonotic agents and food-borne outbreaks in 2016. EFSA J. 2017, 15, 5077. [CrossRef]

2. Humphrey, T.; Jorgensen, F. Pathogens on meat and infection in animals-Establishing a relationship using Campylobacter and Salmonella as examples. Meat Sci. 2006, 74, 89-97. [CrossRef] [PubMed]

3. Obukhovska, O. The Natural Reservoirs of Salmonella Enteritidis in Populations of Wild Birds. Online J. Public Health Inform. 2013, 5, e171. [CrossRef]

4. Hugas, M.; Beloeil, P.A. Controlling Salmonella along the Food Chain in the European Union-Progress over the Last Ten Years. Eurosurveillance 2014, 19, 20804. Available online: http:/ / www.eurosurveillance.org/ViewArticle.aspx?ArticleId=20804 (accessed on 1 April 2020). [CrossRef] [PubMed]

5. Callejon, R.M.; Rodriguez-Naranjo, M.I.; Ubeda, C.; Hornedo-Ortega, R.; Garcia-Parrilla, M.C.; Troncoso, A.M. Reported Foodborne Outbreaks Due to Fresh Produce in the United States and European Union: Trends and Causes. Foodborne Pathog. Dis. 2015, 12, 32-38. [CrossRef] [PubMed]

6. ISO 6579-1:2017; Microbiology of the Food Chain-Horizontal Method for the Detection, Enumeration and Serotyping of Salmonella-Part 1: Detection of Salmonella spp.; International Organisation for Standardisation: Geneva, Switzerland, 2017.

7. Liang, N.; Dong, J.; Luo, L.; Li, Y. Detection of viable Salmonella in lettuce by propidium monoazide real-time PCR. J. Food Sci. 2011, 76, M234-M237. [CrossRef] [PubMed]

8. Lim, H.S.; Zheng, Q.; Miks-Krajnik, M.; Turner, M.; Yuk, H.G. Evaluation of commercial kit based on loop-mediated isothermal amplification for rapid detection of low levels of uninjured and injured Salmonella on duck meat, bean sprouts, and fishballs in Singapore. J. Food Prot. 2015, 78, 1203-1207. [CrossRef] [PubMed]

9. Notomi, T.; Okayama, H.; Masubuchi, H.; Yonekawa, T.; Watanabe, K.; Amino, N.; Hase, T. Loop-mediated isothermal amplification of DNA. Nucleic Acids Res. 2000, 28, e63. [CrossRef] [PubMed]

10. Fratamico, P.M. Comparison of culture, polymerase chain reaction (PCR), TaqMan Salmonella, and Transia Card Salmonella assays for detection of Salmonella spp. in naturally-contaminated ground chicken, ground turkey, and ground beef. Mol. Cell. Probes 2003, 17, 215-221. [CrossRef]

11. Kokkinos, P.A.; Ziros, P.G.; Bellou, M.; Vantarakis, A. Loop-mediated isothermal amplification (LAMP) for the detection of Salmonella in food. Food Anal. Methods 2014, 7, 512-526. [CrossRef] 
12. Wang, D.; Wang, Y.; Xiao, F.; Guo, W.; Zhang, Y.; Wang, A.; Liu, Y. A Comparison of In-House Real-Time LAMP Assays with a Commercial Assay for the Detection of Pathogenic Bacteria. Molecules 2015, 20, 9487-9495. [CrossRef] [PubMed]

13. Nagamine, K.; Hase, T.; Notomi, T. Accelerated reaction by loop-mediated isothermal amplification using loop primers. Mol. Cell. Probes 2002, 16, 223-229. [CrossRef] [PubMed]

14. Hara-Kudo, Y.; Yoshino, M.; Kojima, T.; Ikedo, M. Loop-mediated isothermal amplification for the rapid detection of Salmonella. FEMS Microbiol. Lett. 2005, 253, 155-161. [CrossRef] [PubMed]

15. ISO 16140-2:2016; Microbiology of the Food Chain-Method Validation-Part 2: Protocol for the Validation of Alternative (Proprietary) Methods against a Reference Method; International Organisation for Standardisation: Geneva, Switzerland, 2016.

16. Parida, M.; Posadas, G.; Inoue, S.; Hasebe, F.; Morita, K. Real-time reverse transcription loop-mediated isothermal amplification for rapid detection of West Nile virus. J. Clin. Microbiol. 2004, 42, 257-263. [CrossRef]

17. Francois, P.; Tangomo, M.; Hibbs, J.; Bonetti, E.J.; Boehme, C.C.; Notomi, T.; Perkins, M.D.; Schrenzel, J. Robustness of a loop-mediated isothermal amplification reaction for diagnostic applications. FEMS Immunol. Med. Microbiol. 2011, 62, 41-48. [CrossRef]

18. Tasrip, N.A.; Mokhtar, K.N.; Hanapi, U.K.; Abdul Manaf, A.Y.; Ali, M.E.; Cheah, Y.K.; Mustafa, S.; Mohd Desa, M.N. Loop mediated isothermal amplification; a review on its application and strategy in animal species authentication of meat based food products. Int. Food Res. J. 2019, 26, 1-10.

19. ISO 16140-1:2016; Microbiology of the Food Chain-Method Validation-Part 1: Vocabulary; International Organisation for Standardisation: Geneva, Switzerland, 2016.

20. ISO/TR 6579-3:2014; Microbiology of the Food Chain-Horizontal Method for the Detection, Enumeration and Serotyping of SalmonellaPart 3: Guidelines for Serotyping of Salmonella spp.; International Organisation for Standardisation: Geneva, Switzerland, 2014.

21. Cosby, D.E.; Cox, N.A.; Harrison, M.A.; Wilson, J.L.; Buhr, R.J.; Fedorka-Cray, P.J. Salmonella and antimicrobial resistance in broilers: A review. J. Appl. Poultry Res. 2015, 24, 408-426. [CrossRef]

22. European Food Safety Authority (EFSA); European Centre for Disease Prevention and Control (ECDC). The European Union summary report on trends and sources of zoonoses, zoonotic agents and food-borne outbreaks in 2017. EFSA J. 2018, 16, 262. [CrossRef]

23. Rašeta, M.; Mrdović, B.; Janković, V.; Bečkei, Z.; Lakićević, B.; Vidanović, D.; Polaček, V. Prevalence and antibiotic resistance of Salmonella spp. in meat products, meat preparations and minced meat. In Proceedings of the IOP Conference Series: Earth and Environmental Science, 59th International Meat Industry Conference MEATCON2017, Zlatibor, Serbia, 1-4 October 2017; Volume 85. [CrossRef]

24. Antunes, P.; Mourao, J.; Campos, J.; Peixe, L. Salmonellosis: The role of poultry meat. Clin. Microbiol. Infect. 2016, $22,110-121$. [CrossRef]

25. Nogrady, N.; Kiraly, M.; Davies, R.; Nagy, B. Multidrug resistant clones of Salmonella Infantis of broiler origin in Europe. Int. J. Food Microbiol. 2012, 157, 108-112. [CrossRef] [PubMed] 